\section{Cahiers de Narratologie}

Analyse et théorie narratives

8 | 1997

Création de l'espace et narration littéraire

\title{
La mise en signes de l'espace dans la poésie ibérique après 1965
}

\section{Marie-Claire Zimmermann}

\section{(2) OpenEdition}

1 Journals

Édition électronique

URL : http://journals.openedition.org/narratologie/11745

DOI : 10.4000/narratologie. 11745

ISSN : 1765-307X

Éditeur

LIRCES

\section{Édition imprimée}

Date de publication : 1 décembre 1997

Pagination : 381-395

ISBN : 291089746X

ISSN : 0993-8516

\section{Référence électronique}

Marie-Claire Zimmermann, « La mise en signes de l'espace dans la poésie ibérique après 1965 »,

Cahiers de Narratologie [En ligne], 8 | 1997, mis en ligne le 23 février 2021, consulté le 25 février 2021

URL : http://journals.openedition.org/narratologie/11745; DOI : https://doi.org/10.4000/narratologie. 11745

Ce document a été généré automatiquement le 25 février 2021.

Article L.111-1 du Code de la propriété intellectuelle. 


\title{
La mise en signes de l'espace dans la poésie ibérique après 1965
}

\author{
Marie-Claire Zimmermann
}

1 La création de l'espace en poésie pourrait être un thème de colloque, mais la narration littéraire se trouvant ici au cœur du débat, il parait indispensable d'associer trois paramètres: l'espace, la poésie et le narratif. Je ne me bornerai pas à l'étude de la poésie narrative stricto sensu qui, aux XV ème et XVI ème siècles, a donné en Espagne des romances narrativos et encore au XX ${ }^{\text {ème }}$ siècle la Tierra de Alvargonzàlez d'Antonio Machado et le Romancero gitano de Garcia Lorca : en effet, d'une part, la poésie narrative constitue, à soi seule, presque un mini-genre au sein du genre poétique et, d'autre part, aujourd'hui en Espagne, elle n'est pas pratiquée de manière prédominante. J'ai donc opté pour un corpus poétique où le texte reste fondamentalement un poème, parce qu'il est devenu voix, mais où s'insinue ponctuellement du narratif, sous la forme d'anecdotes, de paraboles, de bribes d'histoires racontées. La voix poématique y adopte donc, parfois, le rôle d'un narrateur, ou du moins engage-t-elle des procédures narratives pour poétiser du fictif, de la fiction, ou pour fictionnaliser le poétique.

2 Nous partirons de quelques observations qui nous serviront d'axiomes méthodologiques. Qu'est-ce qu'un poème sinon un espace occupé par une voix ? Il n'y a pas de poème qui ne soit espace; espace limité sur la page dont les vers, les versets ou les proses phrasées s'achèvent sur du blanc, surface que l'œil contemporain perçoit avec acuité. Espace par essence formel, le poème lyrique célèbre, nomme, représente, objective, matérialise, évoque l'espace, des lieux quels qu'ils soient, abondamment ou non, et il n'est pas de texte, même le plus introspectif, qui ne recoure à l'espace. Et si le locuteur, enfin, se met à raconter, il lui faut situer des personnages, des actions, et là encore surgit un espace pour ces fictions, un espace narrativisé et narrativisant. L'espace est donc définitoire en poésie, forme pour poématiser, contenu de la forme poématique, contenu parfois destiné à la narration mais en ce cas les contenus lyriques et les contenus narratifs peuvent se recouvrir, se confondre pour donner du sens. Il convient, dès lors, d'examiner les procédures selon lesquelles se construit l'espace dans 
le poético-narratif, pour dégager ensuite des significations, une interprétation possible de cet espace créé.

3 Mais l'espace qui est occupé par la voix, pour parler de l'espace et pour le narrer, est tout autant le temps linéaire de la diction, et, nécessairement, l'espace nommé conduit à penser le temps, à le chercher lorsqu'il n'est pas notifié, et le dernier mot du poème est bien un signe d'achèvement où espace et temps s'engloutissent ensemble irrémédiablement. Seule la relecture du texte permettra d'accéder indéfiniment à la représentation et à la signifiance de l'espace poématique, donc encore à celles du temps, mais ceci n'est possible que parce que le texte est un espace inamovible, donné une fois pour toutes.

4 Afin de parvenir à quelques propositions théoriques j'examinerai des œuvres qui se placent à un moment de crise de la poésie espagnole, c'est à dire dans les années 1965-1970, car un tournant esthétique qui est, de fait, une rupture novatrice, permet de saisir les phénomènes d'écriture de manière plus radicale, dans la mesure où le rôle du locuteur (la voix) se trouve majoré et où la question de l'espace/temps y devient nécessairement urgente.

5 Les jeunes écrivains des années 1965-1970 estiment que le modèle poétique prédominant, qui fut en vigueur entre 1950 et 1960 environ, est arrivé à expiration et que l'on ne peut plus fonder le langage poétique sur une démarche protestataire. L'écriture "sociale» a duré à peu près quinze ans: en 1965, elle a atteint ce que j'appelle le seuil de non-création, ceci parce qu'elle s'est uniformisée, banalisée, d'où la nécessité de procéder à des liquidations, qui, toutefois, ne consistent pas à rejeter les grandes œuvres, dites «sociales", telles celles de Bla de Otero, qui sont parvenues à dire l'universel. Les poètes de 1965 ne sont pas moins antifranquistes que leurs prédécesseurs : nés après la guerre civile, ils ne peuvent ni ne veulent parler de ce qu'ils n'ont pas vécu, en revanche tous exècrent l'ennui et le conformisme dans lesquels le franquisme a plongé l'Espagne au cours de ces années de prétendue paix, et ils se lancent à corps perdu dans l'aventure intellectuelle, précisément afin d'échapper à une prostration devenue générale, l'apprentissage de différentes langues constituant notamment l'un des aspects majeurs de leur physionomie culturelle, révélant un même goût pour l'ailleurs, pour la distance, et ces termes impliquent que nous traitons déjà de l'espace réel ou fictif en poésie.

\section{Liquidation du temps historique et de l'espace espagnols.}

6 La langue poétique espagnole ayant eu besoin de se renouveler et de s'ouvrir, vers 1965, une autre esthétique générationnelle a d'abord procédé à l'élimination de l'espace et du temps en rigueur. Ne reprochons pas à ces poètes de ne pas avoir eu souci des procès de Burgos (1970) et de n'avoir que très ponctuellement écrit sur la mort de Salvador Puig Antich (1974) : l'histoire de la poésie espagnole et catalane fait qu'ils ne pouvaient plus fonder langagièrement leur écriture sur une structure spatio-temporelle contraignante. Renonçant donc à cette armature historique ils procèdent à une mise en signes de l'espace radicalement différente dans l'espace du texte poétique. Histoire et espace ayant été étroitement liés, interdépendants, jusqu'en 1965, l'effacement de l'Histoire me paraît être la cause, non la conséquence, de l'irruption de nouveaux espaces en poésie. L'analyse des différentes procédures dont se servent les Novísimos 
montre comment s'élabore un autre système poétique, mais le travail sur l'espace en poésie reconduit toujours inévitablement vers le temps, vers l'Histoire, quels que soient les rejets et les fuites vers une beauté des lieux toute empreinte d'exotisme et d'étrangeté.

\section{Délaissement de l'espace clos pour un espace ouvert}

7 Observons quelques aspects majoritaires de la mutation poétique des années 1965-1970. Après vingt-cinq ans de claustration, les poètes espagnols et catalans sortent de l'espace péninsulaire, à la fois au propre et au figuré. En cette époque de dictature finissante, ils peuvent désormais voyager librement et ils découvrent des pays que leurs lectures poétiques leur ont fait apparaitre comme objets de désir, ainsi l'Angleterre d'Eliot, la France de Mallarmé, l'Allemagne d'Hölderlin et de Rainer Maria Rilke. Le poète espagnol ou catalan de 1965 est un cosmopolite qui prend le train, le bateau, surtout l'avion, mais parfois aussi l'autobus ou la voiture, moins pour fuir l'Espagne que pour aller ailleurs : il n'a aucune raison de s'exiler et il n'est que de passage dans ces pays de plus en plus éloignés de l'Espagne et de l'Europe. Les bornes et les frontières n'interviennent plus dans le discours poétique puisque la démarcation entre la péninsule ibérique et le reste du monde est de moins en moins ressentie historiquement; la fin d'un règne coïncide aussi avec l'ouverture du "royaume». L'espace universel s'ouvre, sans limites, au parcours des mots, alors que le poète engagé ou témoin de son temps demeurait prisonnier d'un espace clos - la prison de Marcos Ana, le quartier pour Gabriel Celaya, le village, la petite ville de province, parfois la métropole, mais en ce cas Barcelone elle-même demeurait enfermée dans des murailles, d'ailleurs depuis longtemps fictives. A cet égard, il n'est que de lire Carlos Barral ou Jaime Gil de Biedma.

Citons quelques recueils qui font date : Arde el mar (1966) de Pere Gimferrer; Dibujo de la muerte (1967) de Guillermo Carnero; Génesis de la luz (1969) et Canon (1973) de Jaime Siles; Sublime solarium (1971) de Luis Antonio de Villena. C'est à partir de ces textes, très significatifs, que l'on étudiera l'accès à une nouvelle mise en signes poétique de l'espace.

Des raisons, d'ordre poétique, font que les Novísimos délaissent l'espace espagnol. En 1970, ces écrivains n'ont plus besoin ni envie de célébrer les paysages espagnols, la terre sèche d'Eugenio de Nora, les déserts de la solitude et de la pauvreté paysannes, parce qu'ils ne cherchent pas à maintenir vivant par la parole ce corps spatial de l'Espagne dont Blas de Otero a nommé les lieux, Avila, Toledo, avec une elliptique et dure ferveur. En 1950, l'espace espagnol était le motif dont on pouvait parler avec lyrisme sans trop encourir les rigueurs de la censure: il servait indirectement de substrat à la remise en cause politique et opérait par délégation pour se redéfinir comme espace maternel, comme patrie réelle, comme source de la langue donc de la poésie, malgré l'Histoire présente. En créant des paysages de mots, les poètes engagés redonnaient forme à une autre Espagne qui se devait donc de couvrir tout l'espace de son territoire. En 1965-1970, les Novísimos se dirigent vers d'autres villes du monde pour y inventer un autre espace poétique, une nouvelle écriture. Ils ne parlent pas d'abord de contrées, de provinces, de paysages ruraux mais de ces cités qui sont des hauts lieux poétiques, des points de départ, où se concentrent les arts, les langages et toutes sortes de cultures. L'espace est donc fondamentalement urbain en poésie. 


\section{Venise} d'ailleurs péjorativement - les jeunes poètes espagnols de 1970 de venecianos et leur esthétique de venecianismo, du moins pendant les toutes premières années d'existence du phénomène novísimo. Ayant voulu célébrer la mémoire de Pound dont les dernières années restent liées à l'espace vénitien, ils s'y rendirent en groupe, en autocar depuis l'Espagne. Les propos antisémites de Pound et son comportement fascisant ne doivent pas nous tromper sur le geste - certes provocateur - des Novísimos, et que l'on n'a pas manqué de leur reprocher. Le châtiment de Pound, sa mise en cage, sont également inacceptables, encore qu'on puisse y percevoir un acte expiatoire ou même rédempteur pour celui qui, enfin, prend peut-être conscience de l'horreur de l'holocauste ; mais surtout, par-delà l'indignité de l'homme Pound, l'œuvre poétique qu'est Cantos transforme la boue en or, constituant un espace créateur au milieu de la désespérance ou de l'indifférence générales. Ni la légitimité d'une cause, ni la souffrance des victimes ne garantissent la valeur du produit poétique qui en est issu, cependant, si le bourreau humilié parvient à accomplir sa tâche poétique - qui dépasse totalement le médiocre destin personnel - celle-ci peut alors devenir le signe d'un rachat collectif et individuel. Le poète demeure libre, même en présence de la barbarie, mais il lui faut l'identifier, l'affronter, non la fuir ; c'est pourquoi il l'explorera, la spatialisera, renouvelant ainsi son propre langage poétique. La Venise de Pound, sous le regard des Novisimos, se pourvoie ainsi d'une multiplicité de signes contradictoires, particulièrement efficaces dans la constitution d'une nouvelle écriture.

11 Le mythe de Venise s'était banalisé à la fin du XIX ${ }^{\text {ème }}$ et tout au long du XX ${ }^{\text {ème }}$ siècle. Dans la mentalité collective, c'est une carte postale, qui après avoir été associée à l'escapade amoureuse loin d'une société répressive (George Sand, Musset) a fini par devenir l'inévitable but de l'officiel voyage de noces ou de l'itinéraire touristique organisé. Cependant, malgré ces connotations négatives, Venise demeure d'abord un espace fabuleux dont la somptuosité et le luxe voisinent avec la moisissure et le pourrissement. Venise tire ses pouvoirs de métaphorisation du fait qu'elle est un double espace: l'un vertical et solide, celui de ses monuments immédiatement identifiés et nommés, l'autre liquide et horizontal, celui des canaux, immortalisés eux aussi par Guardi et Canaletto. Mais ces deux espaces, à leur tour, se rencontrent puisque la surface aqueuse reflète les architectures auxquelles elle renvoie ensuite en une série de jeux vertigineux : la beauté naît paradoxalement de ce qui la détruit, de cette eau qui corrode Venise et qui en fait un kaléidoscope très propice à toute création artistique. Le haut lieu lagunaire suscite le silence de la contemplation d'où jaillissent les voix et les mots, déclenchant ainsi les mécanismes de la fabrication poétique. Les Novisimos avaient besoin d'espaces étrangers ouverts qui favoriseraient et multiplieraient les pouvoirs créateurs du texte. Venise répondait pleinement à leur demande qui était d'abord de nature esthétique.

12 Mais dans quelles structures temporelles pouvaient-ils instaurer l'espace poétique vénitien?

On a souvent écrit que Venise reste a priori liée, dans la mémoire collective, à des artistes de la fin du XIX ${ }^{\text {ème }}$ siècle (Claude Monet) et des années 1900, et que ses détériorations ont aisément nourri le mythe de la décadence et du déclin (Gabriele 
d'Annunzio, Thomas Mann). Les Novísimos semblent se tourner vers le passé, vers une mode rétro, comme si, dans leur recherche d'une beauté métaphorique, ils tenaient à rejoindre les modernistes, qui, eux-mêmes, ont produit leur œuvre à la charnière entre le XIX ${ }^{\text {ème }}$ et le XX ${ }^{\text {ème }}$. La Venise novísima n'est-elle pas un refuge passéiste, un lieu de fuite, où s'opérerait, vers 1970, un curieux transfert de la contemporanéité vers une modernité exclusivement dix-neuvièmiste, c'est à dire finiséculaire? N'est-elle pas le signe, au bout du compte, d'une régression esthétique, alors que ces jeunes gens de vingt ans étaient avides de forger une nouvelle langue poétique en prise sur leur temps?

Il n'est pas facile de répondre à cette question.

Depuis 1880, environ, Venise apparaît comme un espace polysémique, plurisymbolique, tant du point de vue littéraire que plastique. Sa créativité en littérature est autant de nature romanesque que poétique et elle passe aussi par des transpositions cinématographiques. Venise est un lieu idéal pour la fiction. C'est une scène pour un spectacle qui associe la fête et le carnaval, et où l'on a tout loisir de recréer, par une poétique narrative, le lointain passé des Doges. Nous tenons là une des clefs de l'esthétique novísima ; l'espace littéraire de 1970 ne peut plus être cette rue pauvre et triste où erraient les aparecidos de Jaime Gil de Biedma. L'espace doit se démultiplier en de complexes espaces pluriels où se côtoient masques et déguisements, où le bal s'ouvre indéfectiblement sur le défilé funèbre des gondoles conduisant des cercueils vers la lagune. Tant chez Gimferrer que chez Carnero et Villena, la métaphore se fonde sur l'art de la parure et sur le maquillage de la réalité. Cette poétique sert à inventer du nouveau, en se prévalant d'une luxueuse profusion qui n'est pas seulement liée à l'espace visible lui-même, mais qui touche à l'écriture, à ses matériaux et à sa syntaxe.

Alors que les poètes "sociales» n'avaient cessé d'épurer, de simplifier les formes, d'abord pour tenter de rétablir une communication avec leurs interlocuteurs, ensuite pour avancer sur les chemins de la connaissance, les Novísimos optent délibérément pour un style opposé, riche et savant, pour une rhétorique dont les pièces maitresses sont la métaphore - souvent hermétique - l'hyperbate, un lexique rare, des pérégrinismes, l'écriture répondant sans cesse à un souci de distanciation chez le locuteur. Enfin, et ceci est encore plus important du point de vue poétique, l'espace imaginai est généralement en mouvement, traversé de courants et d'itinéraires, à moins que ceux-ci ne coïncident ou ne s'entrecroisent pour produire une dynamique langagière. Ces choix ne s'expliquent pas totalement par le goût de l'art baroque, si souvent revendiqué d'ailleurs par les poètes dans leurs articles critiques et dans leurs essais. Ils ne s'expliquent pas exclusivement non plus par un traitement cinématographique et romanesque de l'espace. Je veux dire par là que le nouveau style des années 1970 ne résulte pas simplement d'une systématique utilisation des arts plastiques en poésie. La mise en signes de l'espace novísimo est liée à une réflexion sur la spécificité de l'écriture poétique: elle ne s'opère que par le biais de ce locuteur anonyme qui est la voix du poème, et qui, en 1970, pour exister, est contrainte de passer par une formulation anachronique et achronique du temps. Le moi, en effet, n'a plus cette continuité, ce désir de logique et de cohérence qui étayaient la difficile démarche d'un Blas de Otero. C'est toute l'histoire des années 1970 qui sous-entend et commande en profondeur cette nouvelle perception poétique de l'espace/temps.

17 Jusqu'en 1960-1965, le locuteur se savait victime d'une dangereuse ankylose. Aussi, pour lutter contre le statisme, devait-il casser le langage, l'abstraire, recourir à 
l'asyndète tout en se situant sur un axe temporel assez clair : par rapport à un passé destructeur, celui de la guerre civile, face à un avenir, celui des lendemains qui chantent, quand l'avènement $\mathrm{du}$ marxisme assurerait le retour à un véritable humanisme. Or, le Novísimo n'a plus envie de parler du passé en poésie et son futur demeure obscur car toutes les idéologies se trouvent ébranlées vers ces années 1968 qui allaient remettre en cause à la fois les pouvoirs, les savoirs et les mœurs. L'écriture novisima marque le rejet de tout subjectivisme et de toute émotion qui seraient liés à l'impact de l'Histoire présente sur le locuteur et ses lecteurs-auditeurs. En revanche, elle s'ouvre à tous les espaces et à tous les temps, tandis que le moi se construit avec toutes ses contradictions à travers le rassemblement des réseaux métaphoriques.

\section{Énonciation et métalangage}

Est-ce à dire que l'énonciateur novísimo est un dandy égotiste qui a échappé à l'Histoire et dont l'objectif n'est que d'élaborer un énigmatique langage incompréhensible pour la inmensa mayoría? Ces écrivains issus de la bourgeoisie aisée s'accommodent-ils des relâchements d'un franquisme presque moribond, pour profiter des plaisirs d'un capitalisme qui s'annoncera vite implacable? Ne se placent-ils pas tacitement audessus de la mêlée pour inventer une écriture culterana, qui se réalise dans l'espace du texte grâce à la polymétrie, au vers blanc, au polysyndète, grâce à un volume phrastique croissant, et, en général, dans un langage en expansion, centripète et non plus centrifuge comme au temps du compromiso? L'espace de la ville mythique, en l'occurrence Venise, apparaît surtout ici comme le signe majeur d'une réflexion métalangagière, comme la preuve d'un travail exigeant, austère et difficile sur le signifiant. La virtuosité technique des Novísimos est manifeste. Tournant le dos au naturel, à l'instinctif, le locuteur explore l'artifice, interrogeant l'envers ou le revers caché des paysages topiques qu'il se propose de transfigurer.

Un texte de Gimferrer dans Arde el mar illustre bien les choix esthétiques des années 1963-1966; il s'agit de Oda a Venecia ante el mar de los Teatros (Poesía, Visor, p. 57-59). L'alliance des termes Venecia et mar se défait vite, pour instaurer, à la place de cette initiale ouverture thématique, un décor, celui d'une scène de théâtre, vide, qui suggère, dès les premiers vers, que tout art est par essence recomposition et que cette Venise gimferrienne n'existe que de biais, par les mots. Le texte, écrit en alejandrinos, fait surgir les motifs d'un ancien voyage du moi adolescent à Venise, mais ceci permet au locuteur de l'ode de signaler ironiquement des émotions qu'il juge dépassées. Une distance s'établit donc entre celui qui commençait à écrire des vers et cet énonciateur qui multiplie les signes spatiaux tous associés au vide et au creux, jusqu'à la dissolution finale dans un miroir qui n'est plus situé à Venise mais en dehors de tout lieu, identifiable seulement dans le présent de l'énonciateur, celui-ci assistant impuissant à l'escamotage de son propre texte.

En rondas de jinetes que disuelve un espejo

negando, con su doble, la realidad de este poema (p. 59)

\section{Ontologie poétique}

20 L'espace vénitien joue dès lors un double rôle, ontologique et méta-langagier à la fois : le moi mesure le passage du temps en inventant des lieux intermédiaires, ces salas del 
recuerdo où la mémoire institue un complexe va-et-vient entre passé et présent, présent et passé. La ville est le lieu où s'accomplit le voyage initiatique du moi, non pas dans l'Histoire présente, mais à travers les prismes et les distances du temps imaginal. Les poètes engagés ne pouvaient se donner le luxe d'entreprendre de tels parcours : leur écriture ne tendait qu'à reconstruire un espace pour une communauté privée de parole. En 1965, le jeune novísimo a tout loisir d'inventer l'espace poétique de son aventure intérieure, sans pour autant céder à la tentation autobiographique. De nouveaux rapports s'établissent ainsi entre le moi et le monde. L'espace novísimo n'est pas décrit et il ne se constitue pas à partir d'énumérations. La ville reste un but à atteindre, telle la Byzance villénienne de Viaje a Bizancío (Poesía, 1970-1984, p. 113-141), ou bien, perdue dans les mots du souvenir, elle devient une source intarissable de langage, la raison d'être de la subversion du temps poétique, donc du maniement de la conjugaison verbale. L'escamotage final de la ville - certes concevable dans le cas de Venise résulte de tours de prestidigitation poétiques : le poème n'existe qu'à ce prix, à travers le sacrifice des images spatiales, toutefois cet élagage n'est efficace que si de multiples signes spatiaux ont été enregistrés en même temps que des motifs ruiniformes. Le langage s'édifie pour se défaire tout en attestant sa finalité poétique. Si les poètes sociaux misaient sur le dénuement formel pour produire plus de sens, les Novísimos minent leurs propres richesses pour parvenir non pas à un sens ou à du sens, mais à une ébauche de signifiance qui contient à elle seule tout le sens, autrement dit l'espace textuel, promis à une perpétuelle exploration formelle.

\section{Espaces de la mémoire : Critique d'une civilisation}

Jusqu'à présent, j'avais toujours pensé que ces espaces poétiques urbains des années 1970, si liés au rêve et à l'imaginaire, ne renvoyaient plus, même indirectement, à cet espace espagnol que les Novísimos avaient éliminé de leur écriture. Or, l'examen approfondi d'un plus grand nombre de textes révèle des signes espagnols sous-jacents qui ne sont jamais innocents et dont l'étude systématique montre que la poésie novísima est beaucoup plus critique qu'on ne l'a dit vis-à-vis des pouvoirs politiques, des pratiques religieuses, des coutumes de l'Espagne des années 1970. L'écrivain ne cherche pas à peindre ou à prouver ; il se sert de procédures très détournées pour faire surgir ces fragments d'espace espagnol qui deviennent extrêmement suggestifs de par leur position dans la structure poématique. C'est dans l'espace, neutre par excellence, de Genève, que le locuteur gimferrien est peu à peu assailli par des bribes de discours anciens qui le ramènent à cette éducation catholique étroite, figée dans des pratiques vides de sens, que lui-même et toute sa génération ont reçue en Espagne. Les mots latins ne sont là que pour créer la dérision. Comment la mémoire se réveille-t-elle de la sorte? Le locuteur parvenant au hasard d'une rue genevoise à la Terrasse Agrippa d'Aubigné (Invocación en Ginebra, id. p. 65-67) ne peut qu'évoquer le vieux poète des Tragiques et les souffrances liées aux guerres de religion. Or, dès le début du texte, les mots oubliés se bousculent dans les versets gimferriens, bien avant que le moi ne nomme l'espace de la Terrasse. Cette Terrasse a-t-elle réellement déclenché la mise en route des souvenirs? Ou ne fait-elle que confirmer cette remontée du passé ? Peu importe. L'habileté poétique consiste ici à subvertir initialement les données temporelles, à produire ce flot chaotique d'un intégrisme religieux qui paraît dérisoire. La Terrasse est l'espace révélateur qui permet au locuteur de comprendre les données 
de sa propre histoire car le calme jardin genevois renvoie, par ressemblance, à ce jardin barcelonais de l'enfance gimferrienne:

se diría

este jardín callado de Ginebra que hoy ostenta tu nombre oh jardín de mis años, oh jardín de mis años y quién sabe

donde mi nombre, Agrippa, mi recuerdo, lo que fui entonces, lo que seré, en qué calle (p. 67)

La relecture de la poésie novísima devra s'effectuer autour de ces jeux de réfraction à travers la mémoire du locuteur, de même aussi en fonction des épigraphes et de divers para-textes où les signes d'Espagne sont là pour projeter un autre éclairage sur les espaces poétiques placés ailleurs, et souvent désignés par des toponymes. Cette tendance s'est beaucoup accentuée depuis les années 1980. Ainsi interprétera-t-on les deux recueils de Julio Llamazares : La lentitud de los bueyes (1979) et Memoria de la nieve (1982) réunis en 1988 chez Hiperión. L'épigraphe du second recueil est un long texte de Strabon qui évoque :

los montañeses, que como dije, son los que habitan en el lado septentrional de Ibería.

Même si les poèmes ne désignent ici que des paysages d'hiver, sans aucune spécificité ibérique, l'on comprend vite que ce livre est une allégorie de la déperdition et de l'occultation du sens en Espagne. Des valeurs profondément liées à l'espace espagnol ont disparu sous la neige symbolique du temps, des civilisations ont été sacrifiées jadis, mais d'autres ensevelissements se produisent maintenant, qui emportent les langages et détruisent les lieux. Ce n'est pas par hasard que Julio Llamazares est né à Vegamian (Léon, 1955), village aujourd'hui disparu sous les eaux d'un barrage.

\section{Catharsis. Langage remis en question. Axiologisation}

Si dans les images de Venise et de Byzance l'on discerne le désir de construire de nouveaux lieux mythiques ou de dire autrement les plus anciens mythes, il n'est pas difficile de déceler sous les métaphores de la mort somptueuse les signes d'une nécessaire catharsis au moment d'inaugurer un autre langage : le locuteur se défait de tous les signes qui ont stérilisé les capacités imaginaires des Espagnols, tant collectives qu'individuelles, durant vingt-cinq années de mythologie franquiste. Pour inventer, le poète doit d'abord oublier des images: Venise et Byzance représentent donc la mise à mort des topiques, mais elles constituent également des allégories de l'acte poétique avec ses renoncements, son obscurité, seuls garants de la production des signes qui vont assurer l'émergence et l'existence du texte.

Nous touchons ici à un autre aspect, à une autre dimension du traitement de l'espace en poésie. C'est dans la mesure où le locuteur l'a très fortement lié à l'Histoire à travers un langage allusif et indirect, non didactique, non moralisateur, que l'espace espagnol ou catalan devient porteur de valeurs universelles, car c'est son inscription dans le poème qui fait accéder la conscience à la notion de limite. En effet, même si les Novísimos ont évacué l'Histoire strictement nationale des années 1970, cela ne signifie pas pour autant qu'ils aient opté pour un idéalisme naïf ou égoïste, ni qu'ils aient misé sur une vision planétaire lénifiante qui garantirait le destin de chacun, sous prétexte d'internationalisation: les plus épicuriens des Novísimos redoutent profondément la vieille barbarie, toujours prête à poindre et à se réinstaller quelque part au monde. 
L'espace poématique demeure donc inscrit sous le sceau de cette menace, et justement par le biais du narratif, car évoquer le sac de Rome en 416 ou le meurtre du roi par Vellido Dolfos aux portes de Zamora revient à dire que l'homme a trop peu changé et que cette évidence doit se glisser inexorablement dans le langage du poète lorsque l'espace y apparaît, à condition toutefois que l'écrivain veuille garder quelque rigueur historique et stylistique. On relira donc les Novísimos en relevant le lexique et les réseaux d'images relatifs à la guerre, à la détention, à la torture, etc. On observera que les textes ont su préserver la mémoire de l'holocauste, y compris par l'évocation d'espaces somptueux où s'insinuent subrepticement des récits ponctués d'images de ruine et de mort, tandis que le locuteur clame l'insuffisance de tout espace poétique face au projet de liquidation totale. Ainsi, un poème de Marcos Ricardo Barnatán portet-il sur le ghetto nuovo de Venise : la ville est ici un emblème efficace qui enserre en ses murs la communauté qui va être sacrifiée, mais par-delà Venise c'est le ghetto de Varsovie qui soudain resurgit, écho nécessaire aux mots du texte, tout aussi violemment inscrit que les noms de Buchenwald et de Dachau dans Cantate en Aleixandre de Gabriel Celaya, poète par excellence de l'engagement dans les années 1950-60.

Cependant, pour rester lisible et viable, l'espace poématique doit poser en termes pluritemporels les questions philosophiques qui déterminent et sous-tendent l'écriture poétique. L'Histoire, qui a servi à instaurer l'espace et qui lui est intégrée, devient ce présent non mesurable où l'homme formule ses doutes et son angoisse face à la mort et à l'anéantissement. La ville n'est plus seulement cet espace où les hommes se rassemblent; elle est le lieu d'où le locuteur, avide d'ubiquité, évoque divers lieux planétaires, surtout ce ciel nocturne qui coïncide avec la méditation ontologique sinon métaphysique. Ce schéma spatial est très courant chez les Novísimos. Citons tout particulièrement les espaces gimferriens inscrits en langue catalane dès 1970, cette Barcelone de L'espai desert (1977) qui, peu à peu, conduit à des espaces vides, ahistoriques, qui deviennent une pure abstraction géométrique de l'espace, c'est à dire l'espace total, la pure virtualité :

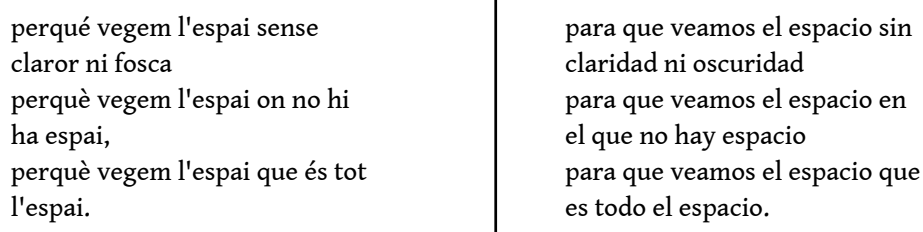

Espejo, espacio y apartantes, Edición bilingüe

Traducción del autor, Visor, Madrid, 1988, p. 206 et 207.

L'espace coïncide avec une structure versale qui a l'allure d'un verset; dépourvue de ponctuation, la phrase instaure un crescendo qui rend compte de l'ampleur de la quête philosophique. L'écrivain procède à des remises en question de l'homme civilisé : il part à la recherche d'autres mythes, tout en construisant de manière critique les formes et les figures d'un moi anonyme, universel, intensément contemporain, avide d'épuiser le langage pour affronter l'espace infini. 


\section{Conclusion}

L'on observe aujourd'hui, dans la poésie écrite dans les deux langues, des phénomènes d'éclatement et de diversification dans le traitement de l'espace, mais aussi des signes de vitalité langagière qui témoignent du tenace désir de retrouver le sens perdu, du besoin de définir une identité, à travers l'écriture de cet espace narrativisé, toujours primordial, qui devient l'introït d'un nouveau temps poétique.

\section{AUTEUR}

\section{MARIE-CLAIRE ZIMMERMANN}

Université de Paris IV-Sorbonne 